\title{
Preoperative Clinical Predictors of Difficult Laryngeal Exposure for Microlaryngoscopy: The Laryngoscore
}

\author{
Cesare Piazza, MD; Stefano Mangili, MD; Francesca Del Bon, MD; Alberto Paderno, MD; \\ Paola Grazioli, MD; Diego Barbieri, MD; Pietro Perotti, MD; Sabrina Garofolo, MD; \\ Piero Nicolai, MD; Giorgio Peretti, MD
}

\begin{abstract}
Objectives/Hypothesis: To identify a clinical predictor score for difficult laryngeal exposure (DLE) during operative microlaryngoscopy.

Study Design: Prospective cohort study in two academic institutions.

Methods: We evaluated 319 patients before microlaryngoscopy for benign and malignant glottic diseases by a standardized preoperative assessment protocol (Laryngoscore) that included 11 parameters: interincisors gap (IIG), thyro-mental distance, upper jaw dental status, trismus, mandibular prognathism, macroglossia, micrognathia, degree of neck flexionextension, history of previous open-neck and/or radiotherapy, Mallampati's modified score, and body mass index (BMI). Each parameter was assessed to obtain a total score. Patients were divided into five classes according to the anterior commissure (AC) visualization: class 0 , complete AC visualization with large-bore laryngoscopes in the Boyce-Jackson position; class I, as class 0 with external laryngeal counterpressure; class II, as class I in the flexion-flexion position; class III, as class II using small-bore laryngoscopes; and class IV, impossible AC visualization.

Results: Class 0-I-II (good/acceptable laryngeal exposure) presented a median score $<6$. This value was chosen as cutoff for distinguishing favorable versus difficult/impossible laryngeal exposures. When the Laryngoscore was $<6$, good laryngeal exposure was observed in $94 \%$ of patients, whereas when $\geq 6$, DLE was encountered in $40 \%$. When considering a Laryngoscore of $\geq 9,67 \%$ of patients had a DLE. At univariate analysis, IIG, upper jaw dental status, macroglossia, micrognathia, degree of neck flexion-extension, and BMI statistically impacted on DLE $(P<0.05)$.

Conclusions: The Laryngoscore is a good predictor of DLE and assists in selecting the ideal candidates for operative microlaryngoscopy.

Key Words: Difficult laryngeal exposure, microlaryngoscopy, transoral microsurgery, glottic tumor, benign glottic lesions.

Level of Evidence: $2 b$.
\end{abstract}

Laryngoscope, 124:2561-2567, 2014

\section{INTRODUCTION}

In the last decades, transoral microsurgery has become a widely accepted surgical procedure for definitive diagnosis and treatment of selected benign and malignant laryngeal diseases. Good exposure and visualization of the glottic plane, especially at the level of the anterior one-third of the vocal cords and anterior commissure (AC), should be always regarded as an essential

From the Department of Otorhinolaryngology-Head and Neck Surgery (C.P., S.M., F.DB., A.P., P.G., DB., P.P., P.N.), University of Brescia, Brescia; and the Department of Otorhinolaryngology-Head and Neck Surgery (s.G., G.P.), University of Genoa, Genoa, Italy 3,2014

Editor's Note: This Manuscript was accepted for publication June

Presented at the 135th Annual Meeting of the American Laryngological Association, Las Vegas, Nevada, U.S.A., May 14-15, 2014.

The authors have no funding, financial relationships, or conflicts of interest to disclose.

Send correspondence to Cesare Piazza, MD, Department of Otorhinolaryngology-Head and Neck Surgery, University of Brescia, Spedali Civili of Brescia, Piazza Spedali Civili 1, 25123 Brescia, Italy. E-mail: ceceplaza@libero.it

DOI: 10.1002/lary.24803 prerequisite for an effective operative microlaryngoscopy. Even though the general principles and techniques of transoral microsurgery have been published in several textbooks and prominent publications, a detailed and comprehensive preoperative flowchart for clinical evaluation and prediction of patient's laryngeal exposure is not universally applied.

Anesthesiologists first defined the concept of the difficult airway and suggested the importance of an accurate preoperative evaluation in order to avoid potential difficulty during orotracheal intubation and reduce the incidence of life-threatening complications. In particular, El-Ganzouri et al. ${ }^{1}$ performed an interesting analysis of several parameters that were able to predict difficult laryngeal visualization before intubation. A thorough review of the pertinent literature allowed us to incorporate a number of different parameters in ElGanzouri's score to obtain a more surgically oriented tool to evaluate the feasibility of transoral microsurgery at the level of the glottic plane.

The aim of this article is to describe this reliable and easily reproducible score (Laryngoscore) to preoperatively detect the occurrence of difficult laryngeal exposure (DLE) before operative microlaryngoscopy. 

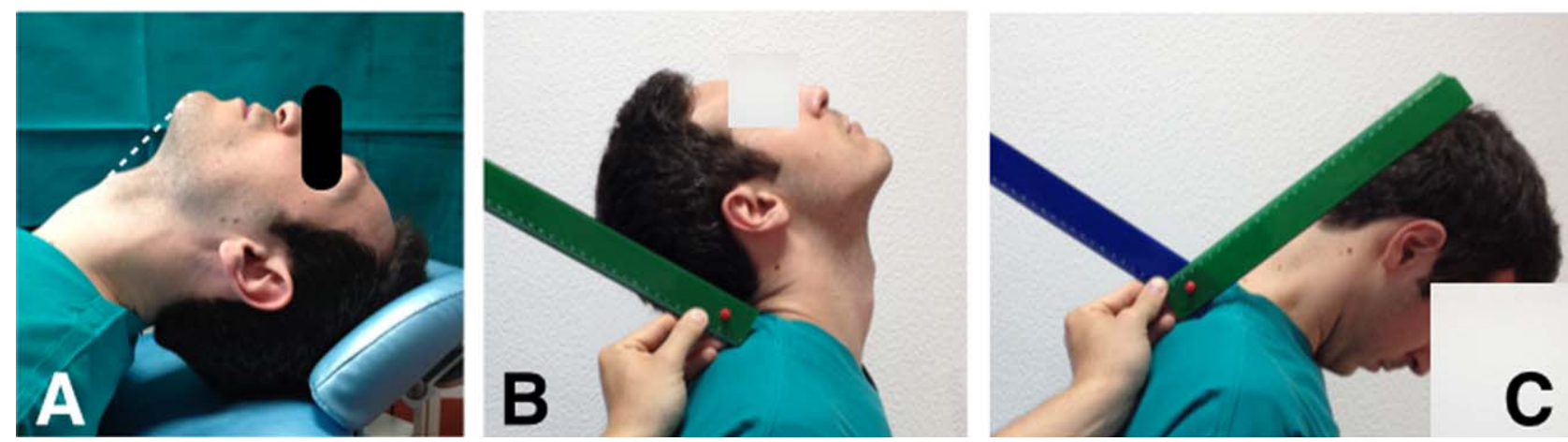

Fig. 1. Measurement of thyro-mental distance along a straight line from the thyroid notch to the lower border of the mandibular symphysis with the head fully extended (A); measurement of the degree of neck flexion-extension by asking the patient to extend the neck (B) and then measuring the arc from this position to that of full flexion of the neck on chest (C). [Color figure can be viewed in the online issue, which is available at www.laryngoscope.com.]

\section{MATERIALS AND METHODS}

After approval was obtained from the institutional review boards of the University of Brescia, Italy, and University of Genoa, Italy, between July 2012 and January 2014 we prospectively evaluated 319 consecutive patients submitted to transoral microsurgery for different glottic diseases (25\% benign lesions, $15 \%$ erythroleukoplakias, and $60 \%$ glottic tumors ranging from T1a to T3). Group A encompassed 163 patients treated at the Department of Otorhinolaryngology-Head and Neck Surgery, University of Brescia, Italy, and group B included 156 patients evaluated and managed at the Department of Otorhinolaryngology-Head and Neck Surgery, University of Genoa, Italy. These two academic institutions share the same diagnostic and treatment philosophy. The entire study population $(\mathrm{N}=319)$ presented a mean age of 63 years (range, 25-100). Patients affected by benign glottic lesions $(\mathrm{N}=80)$ had a mean age of 48 years (range, 25-60), whereas patients affected by precancerous and neoplastic conditions $(\mathrm{N}=239)$ had a mean age of 67 years (range, 39-100).

We standardized a preoperative assessment protocol including 11 parameters: interincisors gap (IIG), thyro-mental distance (TMD), upper jaw dental status, trismus, mandibular prognathism, macroglossia, micrognathia, degree of neck flexion-extension, history of previous open-neck and/or radiotherapy (RT) of the head and neck, Mallampati's modified score, and body mass index (BMI). Each parameter was assessed and scored as follows:

- IIG was measured, with the mouth fully open, between the upper and lower teeth when present, and between the upper and lower gums in edentulous patients. It was then categorized as $\geq 4 \mathrm{~cm}$ (score 0 ) or $<4 \mathrm{~cm}$ (score 1 );

- TMD was measured along a straight line from the thyroid notch to the lower border of the mandibular symphysis with the head fully extended (Fig. 1a), and categorized as $>6.5 \mathrm{~cm}$ (score 0), between 6 and $6.5 \mathrm{~cm}$ (score 1), or $<6 \mathrm{~cm}$ (score 2 );

- Upper jaw dental status was scored 0 in edentulous subjects, 1 in partially edentulous, 2 in presence of normal teeth, and 3 in case of prominent superior teeth;

- Trismus was scored 0 if absent and 1 when present;

- Mandibular prognathism was considered as the position of the lower incisors in front of the upper ones, and was scored 0 when absent or reducible, and 1 when present and not reducible;

- Macroglossia was considered as the situation in which patient's tongue fills the entire oral cavity, and was scored 0 if absent and 1 when present;
- Micrognathia was scored 0 when absent and 1 if present;

- Degree of neck flexion-extension was measured as described by Wilson et al. ${ }^{2}$ by asking the patient to extend the neck and then measuring the arc from this position to that of full flexion of the neck on chest (Fig. 1b, c). The range of motion was categorized as $>90^{\circ}$ (score 0 ), between $80^{\circ}$ and $90^{\circ}$ (score 1 ), or $<80^{\circ}$ (score 2 );

- Previous open-neck surgery and/or RT on the head and neck were scored 0 if never performed and 1 in treated patients;

- Evaluation of oral and oropharyngeal structures was assessed in the sitting position, without phonation, leaving the tongue inside the mouth, by the modified Mallampati's score $^{3}$ assigning score 0 to class I, score 1 to class II, score 2 to class III, and score 3 to class IV;

- BMI was scored 0 when $\leq 25 \mathrm{~kg} / \mathrm{m}^{2}$ and 1 when $>25 \mathrm{~kg} / \mathrm{m}^{2}$ (Table I).

The scores of each parameter were added to obtain a sum potentially ranging from 0 to 17 . The entire Laryngoscore evaluation typically required 3 to 4 minutes to be fully accomplished.

Patients were intubated with a laser-safe endotracheal tube 5.0 or $6.0 \mathrm{~mm}$ in the internal diameter (Laser-Shield; Medtronic Xomed, Jacksonville, FL) under general anesthesia and muscle relaxation by curarization with Mivacurion $2 \mathrm{mg} / \mathrm{ml}$ or Rocuronium Bromide $10 \mathrm{mg} / \mathrm{ml}$. Each institution had a different set of laryngoscopes, essentially distinguished into large-bore for conventional situations (Dedo; Pilling, Philadelphia, PA for group A and Microfrance 121; Microfrance, Paris, France for group B) and small-bore laryngoscopes for unfavorable exposures (Dedo-Ossof; Pilling, Philadelphia, PA for group A and Microfrance 125; Microfrance, Paris, France for group B). The Boston University suspension system (Pilling; Philadelphia, PA) was employed for both groups. ${ }^{4}$ All transoral microsurgical procedures were performed or supervised by one of two experienced laryngologists (C.P. and G.P.).

The first goal for any operative microlaryngoscopy should be that of fully visualizing the entire glottic plane. We therefore chose adequate $\mathrm{AC}$ exposure as an indicator of such an ideal condition. Patients were accordingly subdivided into five categories as follows:

- Class 0 was defined as AC visualization by large-bore laryngoscopes in the Boyce-Jackson position (also called "sniffing" or "flexion-extension" position) ${ }^{5}$ without external laryngeal counterpressure (Fig. 2a, 2b); 
TABLE I.

The 11 Parameters and Related Scoring Systems of the Laryngoscore.

\begin{tabular}{|c|c|}
\hline Parameters & Scores \\
\hline \multicolumn{2}{|l|}{ Interincisors gap } \\
\hline$\geq 4 \mathrm{~cm}$ & 0 \\
\hline$<4 \mathrm{~cm}$ & 1 \\
\hline \multicolumn{2}{|l|}{ Thyro-mental distance } \\
\hline$>6.5 \mathrm{~cm}$ & 0 \\
\hline $6-6.5 \mathrm{~cm}$ & 1 \\
\hline$<6 \mathrm{~cm}$ & 2 \\
\hline \multicolumn{2}{|l|}{ Upper jaw dental status } \\
\hline Edentulous & 0 \\
\hline Partially edentulous & 1 \\
\hline Normal teeth & 2 \\
\hline Prominent teeth & 3 \\
\hline \multicolumn{2}{|l|}{ Trismus } \\
\hline No & 0 \\
\hline Yes & 1 \\
\hline \multicolumn{2}{|l|}{ Mandibular prognathism } \\
\hline Absent or reducible & 0 \\
\hline Present and not reducible & 1 \\
\hline \multicolumn{2}{|l|}{ Macroglossia } \\
\hline No & 0 \\
\hline Yes & 1 \\
\hline \multicolumn{2}{|l|}{ Micrognathia } \\
\hline No & 0 \\
\hline Yes & 1 \\
\hline \multicolumn{2}{|l|}{ Degree of neck flexion-extension } \\
\hline$>90^{\circ}$ & 0 \\
\hline $80^{\circ}-90^{\circ}$ & 1 \\
\hline$<80^{\circ}$ & 2 \\
\hline \multicolumn{2}{|l|}{ Previous treatments (RT, open-neck surgery) } \\
\hline No & 0 \\
\hline Yes & 1 \\
\hline \multicolumn{2}{|l|}{ Mallampati's modified classes } \\
\hline I. Hard and soft palate, uvula, and pillars visible & 0 \\
\hline II. Hard and soft palate, and base of uvula visible & 1 \\
\hline III. Hard and soft palate visible & 2 \\
\hline IV. Only hard palate visible & 3 \\
\hline \multicolumn{2}{|l|}{ BMI } \\
\hline$\leq 25$ & 0 \\
\hline$>25$ & 1 \\
\hline Total & $0-17$ \\
\hline
\end{tabular}

$\mathrm{BMI}=$ body mass index; $\mathrm{RT}$ = radiotherapy.

- Class I was defined as AC visualization by large-bore laryngoscopes in the Boyce-Jackson position with external laryngeal counterpressure ${ }^{6}$ (Fig. 3a, 3b, 3c);

- Class II was defined as AC visualization by large-bore laryngoscopes in the flexion-flexion position ${ }^{6,7}$ with external laryngeal counterpressure (Fig. 4a, 4b);

- Class III was defined as AC visualization by small-bore laryngoscopes in the flexion-flexion position and external laryngeal counterpressure (Fig. 5);
- Class IV was defined as the absolute impossibility to visualize the $\mathrm{AC}$, that is, exposure of the glottic plane limited to its posterior one-third or less, with each of the abovementioned laryngoscopes and maneuvers ${ }^{8}$ (Fig. 6).

We then calculated the median scores obtained in each class and subdivided our cohort of patients into good/acceptable laryngeal exposures (GLE) (classes 0-I-II) and difficult/impossible laryngeal exposures (DLE) (classes III-IV). The median score defining the cutoff between these two conditions was identified to investigate the value of the Laryngoscore as a predictor of GLE versus DLE.

Comparison between the distributions among the five classes of patients in our institutions (group A and B) was performed to assess the reproducibility and interobserver variability of our scoring system.

Specificity, sensitivity, and positive and negative predictive values were then calculated on the entire cohort of patients $(\mathrm{n}=319)$.

Univariate analysis of the impact of each variable on the laryngeal exposure was performed by Stata Release 12 (StataCorp, College Station, TX), setting the threshold of statistical significance at $P<0.05$.

\section{RESULTS}

In group $\mathrm{A}(\mathrm{n}=163), 75(46 \%)$ patients were in class 0 (median score, 2; range, 0-9), 42 (26\%) patients were in class I (median score, 4; range, 0-8), 22 (13\%) patients were in class II (median score, 5; range, 3-11), $21(13 \%)$ patients were in class III (median score, 6; range, $3-11$ ), and only three (2\%) patients were in class IV (median score, 9; range, 3-10).

In group $\mathrm{B}(\mathrm{n}=156), 78(50 \%)$ patients were in class 0 (median score, 3 ; range, 0-9), 41 (26\%) patients were in class I (median score, 3; range, 0-8), 18 (12\%) patients were in class II (median score, 5; range, 3-11), 13 (8\%) patients were in class III (median score, 6; range, 3-11), and six $(4 \%)$ patients were in class IV (median score, 7; range, 3-10). No statistically significant difference was observed in the distribution of patients among different classes between the two institutions.

Because classes 0-I-II (having a GLE) presented median scores $<6$, we chose this value as the cutoff for distinguishing GLE versus DLE. In so doing, when the Laryngoscore value was $<6$, GLE was obtained in $94 \%$ of patients, whereas DLE was found in only $6 \%$ of patients. When the Laryngoscore value was $\geq 6$, we found GLE in $60 \%$ and DLE in $40 \%$ of patients. In particular, when the total score was $\geq 9,67 \%$ of patients presented DLE.

The diagnostic accuracy of the Laryngoscore was calculated on the entire cohort of patients and showed a sensitivity of $63 \%$, specificity of $86 \%$, positive and negative predictive values of $40 \%$ and $94 \%$, respectively.

Univariate analysis found a statistically significant correlation of the following parameters with DLE: IIG $(P<0.001)$, upper jaw dental status $(P<0.001)$, macroglossia $(P=0.002)$, micrognathia $(P=0.002)$, degree of neck flexion-extension $(P=0.016)$, and BMI $(P=0.017)$.

\section{DISCUSSION}

Suspension laryngoscopy and transoral operative microlaryngoscopy are commonly performed for diagnosis 

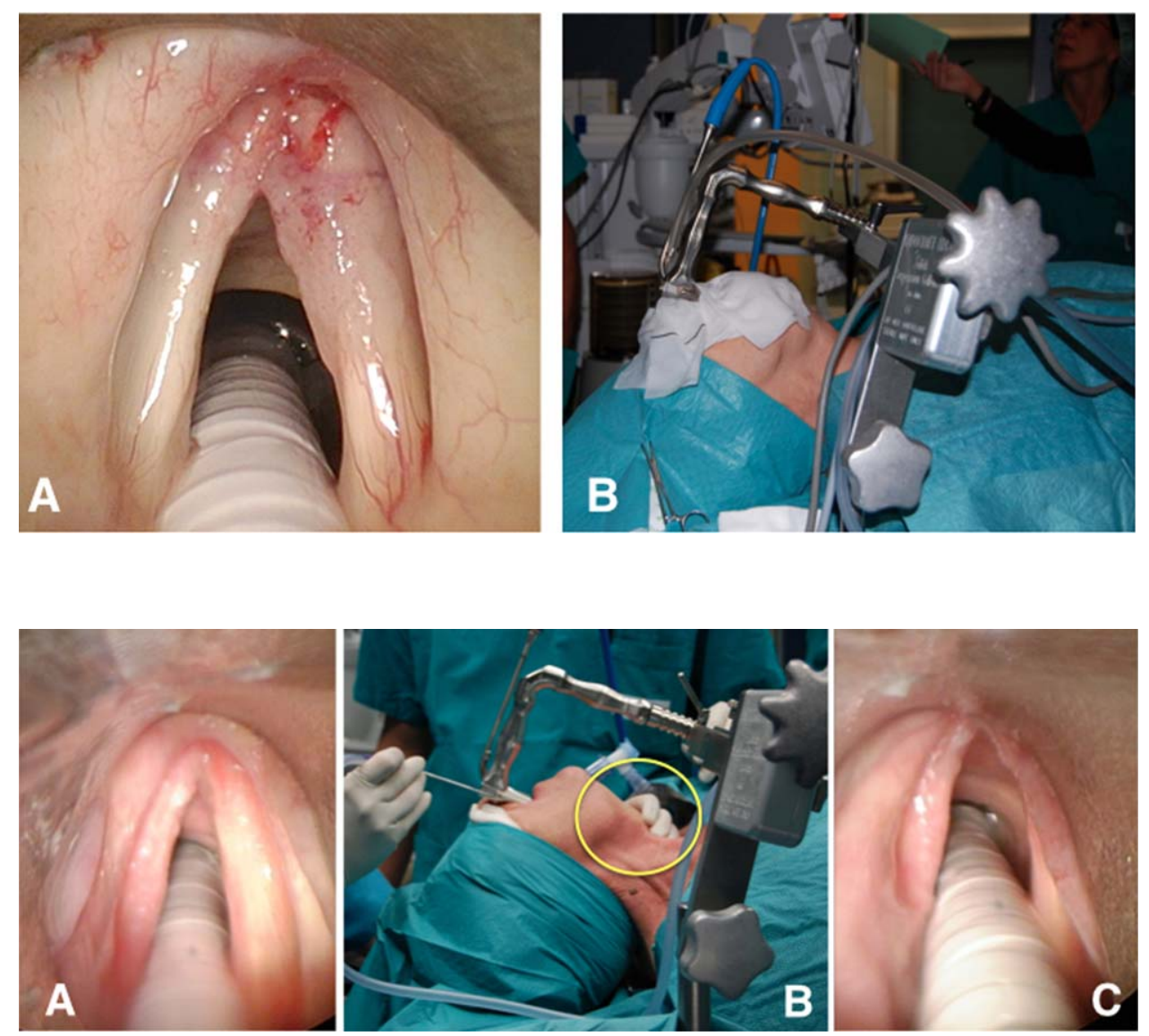

Fig. 3. Class I, or the anterior commissure $(A C)$ visualization, through a large-bore laryngoscope (A) with external laryngeal counterpressure (yellow circle) in the Boyce-Jackson position (B). Note the difference in AC visualization without external laryngeal counterpressure (C). [Color figure can be viewed in the online issue, which is available at www.laryngoscope.com.]

and treatment of laryngeal lesions. The importance of intercalating large-bore laryngoscopes to visualize the entire glottic plane, allowing binocular vision and bimanual working in such a clinical scenario, cannot be overemphasized. Although to the best of our knowledge no systematic attempt has ever been performed to correlate the degree of laryngeal visualization with oncologic results for specific $\mathrm{T}$ categories, or to the functional outcomes after phonosurgical procedures for benign diseases, it is intuitive that the ideal condition for treating such clinical problems should be adequately assessed during preoperative workup. In fact, if the entire glottis is not appropriately visualized through direct laryngoscopy due to suboptimal laryngeal exposure, misdiagnosis, incomplete resection, or inadvertent injury to teeth and pharyngolaryngeal soft tissues can be easily encountered. ${ }^{7}$ Moreover, the preoperative assessment of adequate laryngeal exposure is of paramount value both in benign and
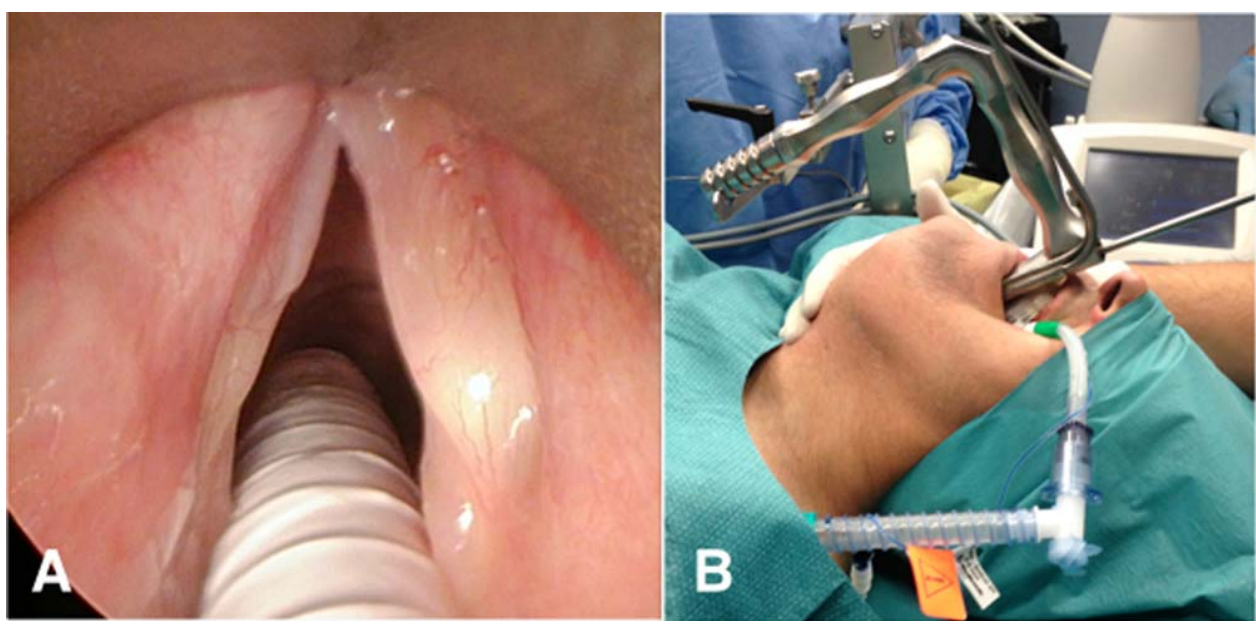

Fig. 4. Class II, or the anterior commissure visualization, through a large-bore laryngoscope (A) with external laryngeal counterpressure in the flexion-flexion position (B). [Color figure can be viewed in the online issue, which is available at www.laryngoscope.com.] 


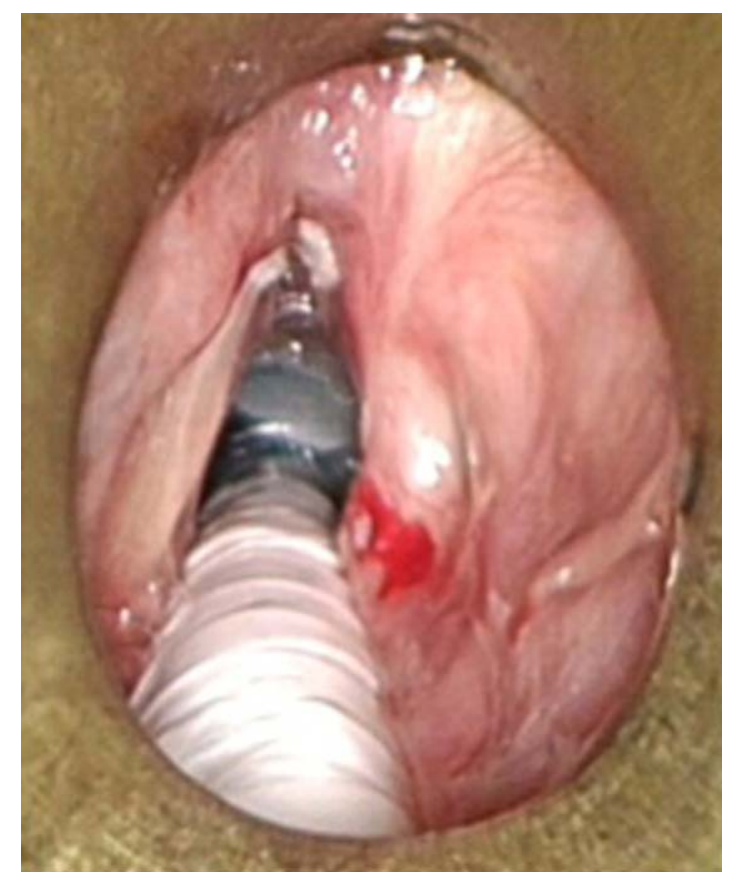

Fig. 5. Class III, or the anterior commissure visualization, through a small-bore laryngoscope with external laryngeal counterpressure in the flexion-flexion position. [Color figure can be viewed in the online issue, which is available at www.laryngoscope.com.]

preneoplastic/neoplastic conditions. In routine phonosurgical procedures, for example, DLE should prompt the novice to seek the assistance of more expert colleagues. On the other hand, in the oncologic setting, DLE in the presence of a small T1a of the midcord may be overcome by adequate experience, whereas the same condition in case of $\mathrm{T} 1 \mathrm{~b}$ involving the anterior commissure or bulky $\mathrm{T} 2$ could require other treatment strategies (RT or open-neck procedures).

The incidence of DLE and difficult intubation (somewhat related each other) is reported to range between $1.5 \%$ to $24 \%$ and $1 \%$ to $4 \%$, respectively. ${ }^{9,10}$ These data are confirmed by our series, where we found $10.7 \%$ of difficult (class III) laryngeal exposures and $2.8 \%$ of impossible (class IV) laryngeal exposures for surgical purposes different from a simple diagnostic endoscopy or biopsy. This means that in around $14 \%$ of our patients, therapeutic operative microlaryngoscopy for "difficult" lesions (i.e., T1a of the anterior one-third of the vocal cord, T1b involving the AC, and T2-T3 glottic diseases) may have been performed in suboptimal (or impossible) conditions. This observation stresses the importance of adequate preoperative evaluation and possible prediction of DLE before each operative microlaryngeal procedure, especially in the oncologic setting.

In the recent literature, only a few reports have attempted to address the preoperative predictors of DLE. A short, stiff, and muscular neck; macroglossia; retrognathia; obesity; and various anatomical conditions such as limited extension of the cervical spine have been suggested as possible causes of DLE. However, these parameters alone have shown a lack of consistency in predicting DLE, and considerable disagreement exists about which parameters are the most valuable for this aim. For example, Hsiung et al. ${ }^{8}$ claimed that the combination of gender and thyroid-mandible angle (TMA), in their experience, provided an important clinical indicator for DLE. They assumed that men are more prone to have a muscular neck and stiff larynx and noticed that the best cutoff point for TMA is $120^{\circ}$ for males and $130^{\circ}$ for females. However, we did not include TMA in our Laryngoscore because we felt it was not easily evaluable during routine preoperative clinical assessment, whereas we aimed toward a practical and reproducible tool.

Pinar et al. ${ }^{9}$ investigated potential parameters to identify DLE and by multivariate analysis found that neck circumference $(>40 \mathrm{~cm})$, hyoid-mental distance $(<$ $6.05 \mathrm{~cm})$, and sternum-mental distance $(<13.9 \mathrm{~cm})$ were statistically significant predictors for this condition. Herein, patients who received previous treatments (surgery, RT) or trauma on the neck were excluded from the analysis. By contrast, we decided to include the history of previous open-neck surgery and/or RT on the head and neck as a variable in our score due to the paramount influence played, in our experience, by iatrogenic soft tissues sclerosis and fibrosis on the feasibility of suspension laryngoscopy. However, we avoided neck circumference by using BMI as its surrogate and substituted sternum-mental and hyoid-mental distances with the thyro-mental one, which is easier to measure.

Roh and Lee ${ }^{11}$ analyzed 85 patients submitted to microlaryngoscopy, confirming that obesity, short neck, and retrognathia can be used as preoperative clinical predictors for DLE. This study has two important biases, however: exclusion criteria (absence of upper incisors, history of previous treatments on the neck, tempomandibular joint disorders, facial anomalies) and the fact that external laryngeal counterpressure was not considered because it could show individual variations and be a confounding variable in the prediction of DLE. In our prospective evaluation, no exclusion criteria were adopted in order to make it more similar to the daily life requirements of a busy laryngologic service: all

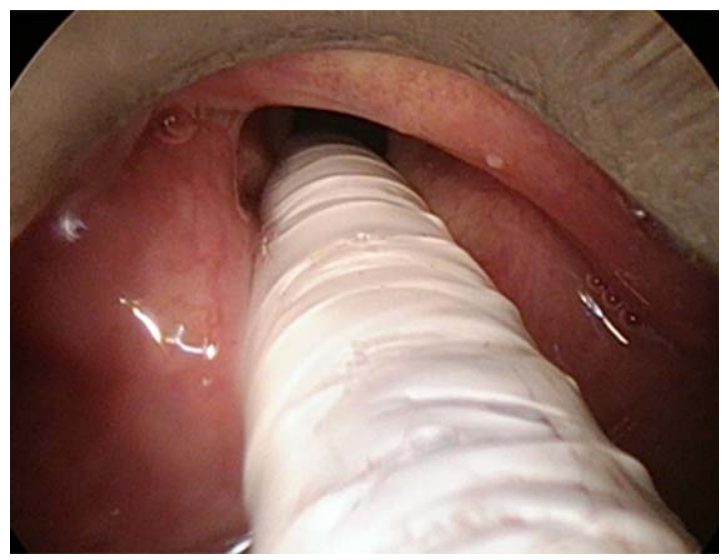

Fig. 6. Class IV, or impossible anterior commissure visualization with any type of laryngoscope or position. [Color figure can be viewed in the online issue, which is available at www.laryngoscope.com.] 
candidates for operative microlaryngoscopy were thus preoperatively evaluated by the Laryngoscore. Moreover, we also deliberately included in the present study patients who were affected by benign glottic diseases (with a mean age younger than usually observed in the oncologic setting) in order to confirm the possibility to also extend the Laryngoscore's findings to a nonneoplastic, usually healthier population.

As mentioned before, we empirically chose 6 as the cutoff score to have a high negative predictive value. In fact, when the score was $<6$, we observed GLE in $94 \%$ of patients; therefore, this situation can be considered the ideal for treating "difficult" lesions and teaching operative microlaryngoscopy to residents and junior staff members. Conversely, when the Laryngoscore was $\geq 6$, $40 \%$ of patients had DLE, an aspect that should be always discussed with patients during preoperative counseling. Furthermore, the percentage of DLE increased to $67 \%$ when a score $\geq 9$ was encountered.

Concerning the impact of different parameters in predicting DLE, we performed a subanalysis of class III $(\mathrm{n}=34)$ for which teeth status was scored $>1$ in $94 \%$ of cases, modified Mallampati's score was $>2$ in 59\%, ability to extend the neck was $<90^{\circ}$ in $56 \%$, IIG was $<4 \mathrm{~cm}$, and TMD $<6.5 \mathrm{~cm}$ in $40 \%$ of patients each. In class IV $(\mathrm{n}=9)$, a teeth score $>2$ was observed in $100 \%$ of patients, modified Mallampati's score was $>2$ in $78 \%$, and the IIG was $<4 \mathrm{~cm}$ in $67 \%$, whereas the ability to extend the neck $<90^{\circ}$ and TMD $<6.5 \mathrm{~cm}$ were observed in $56 \%$ of patients each. These findings were also confirmed by univariate analysis where a reduced IIG, unfavorable upper jaw dental status, macroglossia, micrognathia, limited degree of neck flexion-extension, and high BMI have a statistically significant impact on DLE. As a result, apart from the absolute value of the total score, the presence of such specific negative predictors should always alert the surgeon about a higher chance of having DLE. However, we maintained all 11 variables in our Laryngoscore because $59 \%$ of patients in class III and $75 \%$ of those in class IV had unfavorable conditions in at least one of the statistically not significant variables tested. Therefore, the apparent redundancy of the present score seems justified to correctly predict DLE in a subset of patients with alterations of both statistically significant and not significant variables. Future studies with larger populations will be possibly able to discover a significant impact on DLE of other variables (such as previous treatments or trismus) included in our score system but rarely encountered.

Friedrich and Gugatschka ${ }^{12}$ quantified the forces occurring during fulcrum-based laryngoscopy and found that elevating the patient's head by flexing the cervical spine (in the flexion-flexion position) significantly reduced the force to obtain adequate $\mathrm{AC}$ exposure. In a recent article, Tong et al. ${ }^{13}$ investigated the influence of head and neck position during 14 microlaryngoscopies using MRI and reinforced the concept that flexionextension is the optimal position for microlaryngoscopy. Accordingly, all patients evaluated in our prospective study started maneuvers for suspension laryngoscopy in the Boyce-Jackson position, and only when external counterpressure was insufficient to obtain adequate $\mathrm{AC}$ visualization did we adopt the flexion-flexion position (class II) and tilt the operating table in a Trendelenburg position.

For the management of DLE, Ohno et al. ${ }^{10}$ evaluated 212 patients (mainly affected by benign lesions), performing operative microsurgery in the BoyceJackson position and using triangular-shaped laryngoscopes for laryngeal exposure. In case of DLE $(6.6 \%$ of their series), posture and laryngoscope were modified as needed to adequately expose the target lesion. If these changes were not sufficient to obtain adequate visualization of the glottic plane, the authors switched to fiberoptic laryngeal surgery under local anesthesia. In another report, Cheng and $\mathrm{Woo}^{14}$ recently proposed an interesting rescue strategy when unexpected DLE was encountered. They proposed to switch from a small orotracheal tube to jet ventilation when possible to improve the working area by using the smallest size of the jetting catheter $(3 \mathrm{~mm})$. This maneuver allows for preserving binocular vision and bimanual tissue manipulation. However, protection of the distal airway is sacrificed, and some slight movements of the operative field with each pulse of the jet ventilation can occur. When this strategy fails, long, angled, rigid telescopes and microlaryngeal instrumentation may be used. If an assistant is available to hold the telescope, the surgeon may be able to apply bimanual tissue manipulation. Finally, in patients who cannot be orotracheally intubated, a laryngeal mask anesthesia (LMA) device can be used to ventilate and treat the patient. By placing the LMA over the vestibule, in fact, the patient can be ventilated while a fiberoptic laryngoscope with a biopsy channel is inserted for removal of the target lesion. This technique allows general anesthesia to be used for the procedure, but only limited tissue sampling can be obtained with the single-channel instrumentation. Moreover, there is no stable operating platform, and the ability to control bleeding is limited. However, it is clear that with each of the abovementioned escalations, a parallel degradation of the advantages afforded by traditional operative laryngeal microsurgery occurs. In each case, anticipation of such technical difficulties by the Laryngoscore during preoperative workup may be of great assistance in reducing the amount of stress, uncertainty, and intraoperative problems encountered. In fact, better surgeon-patient and surgeon-anesthesiologist counseling are two of the most important outcomes expected from the routine application of such a clinical tool.

\section{CONCLUSION}

The Laryngoscore herein presented is easy to use, reliable, and reproducible. It allows identifying GLE with a great confidence, while alarming about possible DLE (especially in the presence of high scores and alteration of specific parameters). In our experience, its role has been of paramount importance in patient selection, treatment planning, evaluation of alternative therapeutic options, and to teach microlaryngoscopy to the residents. 


\section{BIBLIOGRAPHY}

1. El-Ganzouri AR, McCarthy RJ, Tuman KJ, Tanck EN, Ivankovich AD. Preoperative airway assessment: predictive value of a multivariate risk index. Anesth Analg 1996;82:1197-1204.

2. Wilson ME, Spiegelhalter D, Robertson JA, Lesser P. Predicting difficult intubation. Br J Anaesth 1988;61:211-216.

3. Mallampati SR, Gatt SP, Gugino LD, Desai SP, Waraksa B, Freiberger D, Liu PL. A clinical sign to predict difficult tracheal intubation: a prospective study. Can Anaesth Soc J 1985;32:429-434.

4. Zeitels SM, Burns JA, Dailey SH. Suspension laryngoscopy revisited. Ann Otol Rhinol Laryngol 2004;113:16-22.

5. Jackson C, Jackson CL. Cancer of the Larynx. Philadelphia, PA: WB Saunders; 1939: 17-18.

6. Zeitels SM, Vaughan CW. External counterpressure and internal distension for optimal laryngoscopic exposure of the anterior glottal commissure. Ann Otol Rhinol Laryngol 1994;103:669-675.

7. Hochman II, Zeitels SM, Heaton JT. Analysis of the forces and position required for direct laryngoscopic exposure of the anterior vocal folds. Ann Otol Rhinol Laryngol 1999;108:715-724.
8. Hsiung M-W, Pai L, Kang B-H, Wang B-L, Wong C-S, Wang H-W. Clinica predictors of difficult laryngeal exposure, Laryngoscope 2004;114:358 363

9. Pinar E, Calli C, Oncet S, Selek B, Tatar B. Preoperative clinical prediction of difficult laryngeal exposure in suspension laryngoscopy. Eur Arch Otorhinolaryngol 2009;266:699-703.

10. Ohno S, Hirano S, Tateya I, Kojima T, Ito J. Management of vocal fold lesions in difficult laryngeal exposure in patients in phonomicrosurgery. Auris Nasus Larynx 2011;38:373-380.

11. Roh J-L, Lee Y-W. Prediction of difficult laryngeal exposure in patients undergoing microlaryngosurgery. Ann Otol Rhinol Laryngol 2005;114: 614-620.

12. Friedrich G, Gugatschka M. Influence of head positioning on the forces occurring during microlaryngoscopy. Eur Arch Otorhinolaryngol 2009 266:999-1003.

13. Tong B, Fang R, Smith BL. Study of the head and neck position in microlaryngoscopy using magnetic resonance imaging. Eur Arch Otohinolaryngol 2013;270:243-247.

14. Cheng J, Woo P. Rescue microlaryngoscopy: a protocol for utilization of four techniques in overcoming challenging exposure in microlaryngeal surgery. $J$ Voice 2012;26:590-595. 